\title{
Relaciones de redes, perspectiva de internacionalización para la pyme Gastro Innova
}

\author{
Maria Merced Montenegro-Arcila ${ }^{1}$ \\ Fundación Universitaria de la Cámara de Comercio de Bogotá -Uniempresarial \\ mmontenegro@ue.edu.co
}

DOI: https://doi.org/10.21158/23227230.v10.n0.2020.2841

Cómo citar este artículo: Montenegro-Arcila, M. M. (2020). Relaciones de redes, perspectiva de internacionalización para la pyme Gastro Innova. Revista Ploutos, 10, (Páginas). DOI: https://doi.org/10.21158/23227230.v10.n0.2020.2841

Fecha de recepción: 21 de agosto de 2020

Fecha de aprobación: 20 de octubre de 2020

\section{Resumen}

Las teorías de internacionalización explican cómo las empresas desarrollan sus procesos para la exportación de productos y servicios, y cómo el impacto de las relaciones de redes los dinamiza con miras a superar algunas limitantes. Por esta razón, este trabajo tiene como objetivo situar las relaciones de redes en modos de entrada al mercado internacional. Al explorar tres casos de estudio de empresas colombianas, se describen brevemente las estrategias y los factores que aceleraron el proceso y cómo la consolidación de las relaciones sociales y comerciales impacta su internacionalización. De esta manera, al analizar desde la teoría de redes se puede suscitar, como un modo de entrada de alto grado, la perspectiva de internacionalización para la pyme Gastro Innova. Se tienen en cuenta tres perspectivas teóricas de internacionalización que servirán en el propósito de apoyar el análisis del crecimiento de una empresa a nivel internacional. Primero, se abordan temas como tipos de estrategias y factores claves para la internacionalización, posteriormente, se mencionan algunos tratados internacionales vigentes que colaboran en el fortalecimiento de las relaciones macroeconómicas y, por último, se trata el impacto que tienen las relaciones de redes para la internacionalización de las pymes.

Palabras clave: internacionalización; internacionalización de pymes; teorías de internacionalización; relaciones de redes; mercado internacional; relaciones comerciales; relaciones macroeconómicas.

\footnotetext{
${ }^{1}$ Profesional en Negocios Internacionales - Fundación universitaria Uniempresarial de la Cámara de Comercio de Bogotá. Como estudiante participo en el programa de semillero de investigación "Internacionalización de Empresas" su trabajo se centra específicamente en la perspectiva de internacionalización de pymes. ORCID: https://orcid.org/0000-0003-2491-3480 


\section{Network relations, \\ a perspective to the internationalization of the SME Gastro Innova}

\section{Abstract}

The theories of internationalization explain how firms develop their processes for exporting products and services, and how the impact of network relations invigorates them towards overcoming some constraints. For this reason, this paper aims at putting network relations into international market entry modes. As a result of exploring three case studies of Colombian companies, we briefly describe the strategies and factors that sped up the process, and how the consolidation of social and business relations impacts their internationalization. In this way, the perspective to the internationalization of the SME Gastro Innova can be raised as a high-level entry mode when analyzed from the network theory. Three theoretical perspectives of internationalization that will serve the purpose of supporting the analysis of the growth of a company at the international level are considered. First, topics such as types of strategies and key factors for internationalization are addressed; after that, some current international treaties that collaborate in the strengthening of macroeconomic relations are mentioned; and finally, the impact of network relations for the internationalization of SMEs is discussed.

Keywords: internationalization; SME internationalization; internationalization theories; network relations; international market; business relations; macroeconomic relations.

\section{Relações de redes, \\ perspectiva de internacionalização para a PME Gastro Innova}

\section{Resumo}

As teorias de internacionalização explicam como as empresas desenvolvem seus processos de exportação de produtos e serviços e como o impacto das relações de redes as fortalece visando superar algumas limitações. Por esse motivo, este trabalho tem como objetivo localizar as relações de redes nas formas de inserção no mercado internacional. Ao explorar três estudos de caso de empresas colombianas, são brevemente descritos as estratégias $e$ os fatores que aceleraram o processo e como a consolidação das relações sociais e comerciais impacta sua internacionalização. Dessa forma, ao analisar a partir da teoria das redes, a perspectiva de internacionalização da PME Gastro Innova pode ser levantada como uma modalidade de entrada de alto grau. São consideradas três perspectivas teóricas de internacionalização que servirão de suporte à análise do crescimento de uma empresa internacional. Primeiramente são abordados temas como tipos de estratégias e fatores-chave para a internacionalização, posteriormente, são mencionados alguns tratados internacionais vigentes que colaboram para o fortalecimento das relações macroeconômicas e, finalmente, menciona-se o impacto que as relações em rede têm para a internacionalização das PME.

Palavras-chave: internacionalização; internacionalização de PME, teorias de internacionalização, relações de redes, mercado internacional, relações comerciais, relações macroeconómicas. 


\author{
Réseaux, relations \\ et perspectives d'internationalisation de la PME Gastro Innova
}

\begin{abstract}
Résumé
Les théories de l'internationalisation explicitent la façon dont les entreprises développent leurs processus d'exportation de biens et services, et mettent en lumière la dynamisation et l'impact positif des réseaux et relations pour surmonter certaines difficultés. Cette étude vise à comprendre comment les relations et réseaux peuvent permettre l'incursion des PME sur les marchés internationaux. Nous décrirons succinctement les stratégies et facteurs d'accélération du processus et l'impact de la consolidation des relations sociales et commerciales sur l'internationalisation des PME. L'analyse théorique des réseaux de relations permettra d'expliquer le processus d'internationalisation de la PME Gastro Innova selon la prise en compte de trois perspectives qui serviront à l'étude de la croissance internationale de cette PME. Nous aborderons d'abord les types de stratégies et facteurs clés d'internationalisation avant de nous intéresser à certains traités internationaux pour la collaboration et le renforcement des relations économiques et finaliserons par l'étude d'impact des réseaux de relations sur l'internationalisation des PME.
\end{abstract}

Mots-clés: internationalisation; internationalisation des PME; théories de l'internationalisation; réseaux de relations; marché international; relations commerciales; relations macroéconomiques.

\title{
1. Introducción
}

El presente artículo de investigación cualitativa busca concienciar el impacto que tiene la construcción de relaciones sociales y comerciales de bajo contexto cultural y globalizadas (Bauman, 2016; Johanson y Wiedersheim-Paul, 1975) en relación con la internacionalización de empresas y con un enfoque en la perspectiva teórica de redes como un modo de entrada al mercado externo para empresas colombianas en desarrollo y abiertas a los cambios de la economía, como es el caso de Gastro Innova, una pyme que elabora productos alimenticios.

En el inicio, el sector de alimentos procesados listos para consumir estableció una serie de fortalezas entre los consumidores de alimentos tipo gourmet; sin embargo, estas empresas no cuentan con la orientación suficiente para ejecutar el plan de internacionalización y su relacionamiento de redes es bajo (Procomer, 2017). El fuerte uso de redes facilita la toma de decisiones en la selección del mercado y en el modo de entrada al terreno internacional (Johanson y Vahlne, 1977 
Aunque en los últimos diez años se ha notado un importante interés en el cambio de mentalidad por parte de los gerentes de pymes, todavía existen deficiencias en la dirección de las empresas colombianas (Cardozo, Chavarro y Ramírez, 2013). Del mismo modo, en el foro «Encuentro por la Transformación Productiva e Internacional de las Pymes» (Propaís, 2009), se evidenció que el mercado en el que operan las pequeñas y medianas empresas no exige especialización y estándares de calidad altos, lo cual provoca poca innovación y bajo crecimiento y repercute en el abastecimiento de insumos, en el acceso a las cadenas de valor y en el desarrollo de la comercialización de productos y servicios.

Así mismo, depender de mercados afectó a los sectores; en el caso del sector de alimentos procesados, se evidenció una disminución significativa en el 2009, del 3,5\%, en las exportaciones a Venezuela y Ecuador por el descuido en las relaciones diplomáticas (Aladi, 2011).

Luego de la depuración del Registro Único Empresarial y Social (RUES) que ordenó la Ley 1727 de 2014, que se realizó en 2015, en 2016 se identificó que el número anual de empresas liquidadas se estabilizó alrededor de las 21 mil empresas. Las empresas canceladas fueron en su mayoría microempresas dedicadas al comercio -36\%-, alojamiento y servicios de comida -14\%- e industrias manufactureras -10\%-. (Cámara de Comercio de Bogotá, 2009)

Por consiguiente, la problemática ha hecho que las pymes identifiquen nuevos modos para administrar los negocios a fin de que la gobernanza, la tecnología, el transporte y la capacidad de producción tengan viabilidad (Puerto-Becerra, 2010).

Dicho lo anterior, en los procesos y las exigencias de la globalización económica (Drucker, 1993) es necesario el conocimiento experiencial que aporte a la perspectiva del emprendimiento de internacionalización y a la construcción de las relaciones de redes que favorezcan la entrada al mercado internacional (Irineo, Morales, Mora y Montemayor, 2015).

En pocas palabras, con miras al crecimiento empresarial internacional de los principales generadores de empleo, como lo son las pymes (Amestoy, 2009), la estrategia de internacionalización se debe plantear desde los recursos de los que dispongan (Knight y Cavusgil, 2004), en un mundo que se revela como diverso en el entorno económico y 
regulatorio (Rojas-Villa, 2015), y en donde muchas personas empiezan a descubrir y luego a enamorarse y valorar o incorporar en la vida costumbres de otros pueblos (Giddes, 2000).

Por consiguiente, se debe pensar todo el tiempo en cómo articular la cadena de valor (Viscarri-Colomer, 2011) a través de relaciones sociales y comerciales que generen la entrada a una audiencia mucho más democrática (Hernández-Pereira, 2017). Esta premisa nos permitirá responder a la siguiente pregunta de investigación: ¿por qué las relaciones sociales y comerciales impactan la perspectiva y el proceso de internacionalización de las pymes?

Así mismo, se plantea como objetivo general situar la consecución de relaciones de redes en modos de entrada para la internacionalización de pymes.

Como objetivos específicos se establecen los siguientes:

- Mencionar algunas teorías de internacionalización y los factores que influyen en las transacciones de mercados internacionales.

- Describir los tipos de estrategias de internacionalización que utilizan las empresas estudiadas y el impacto que tienen las relaciones de redes en la selección del mercado y el modo de entrada.

\section{Marco teórico}

\subsection{Estudio de las teorías y los factores de internacionalización}

Se presentan tres perspectivas teóricas sobre la internacionalización, con un análisis que se plantea desde temas relevantes como modo de entrada al país destino y la selección del mercado en el contexto de la globalización económica. Cada una de las perspectivas teóricas involucra momentos y factores que influyen en las operaciones extranjeras de las empresas, la de Uppsala, la de ventaja competitiva y la de redes proporcionan elementos notables, aunque se hace necesario compararlas. 
Figura 1. Globalización económica

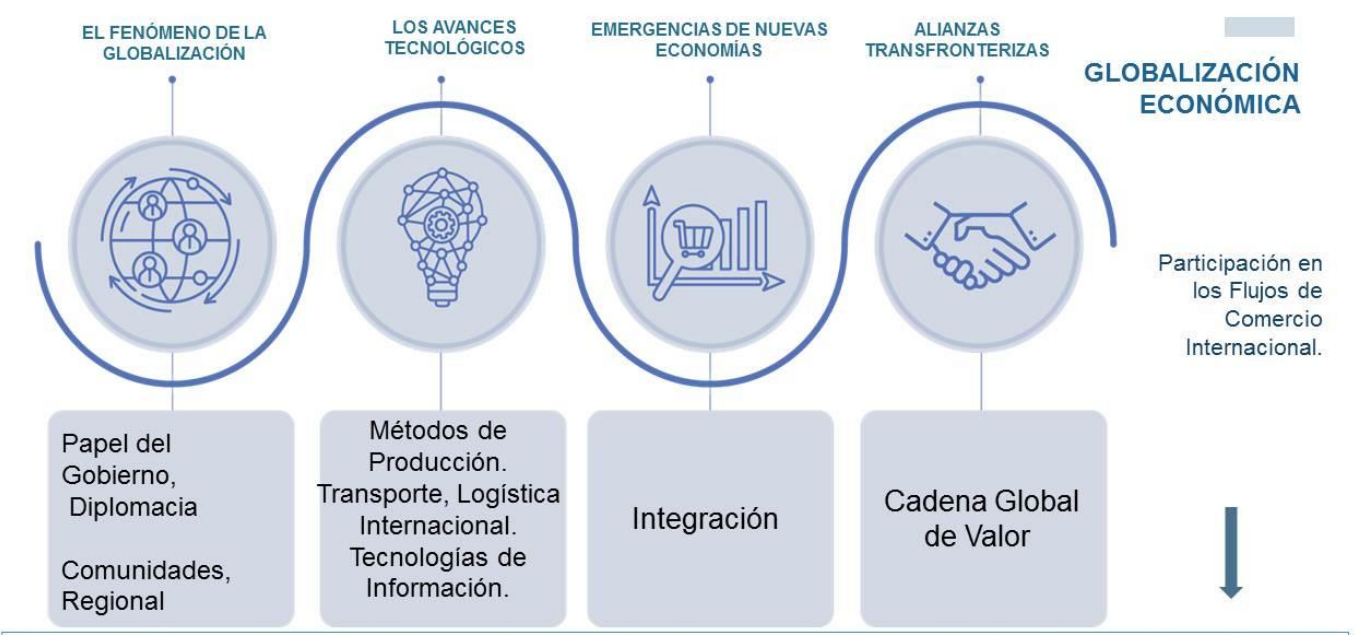

Fuente. Elaboración propia.

Existen empresas que en los procesos de internacionalización sopesan factores como los que muestra la figura 1, nuevas condiciones del mercado en muchos sectores de la economía, redes globales crecientes, transporte, la tecnología de punta en las áreas de producción, las comunicaciones, las habilidades y la experiencia del emprendedor. Estos elementos determinantes llevan a un alto grado de internacionalización, incluso lo aceleran antes de que nazca la empresa, como es el caso de Born Global (Knight y Cavusgil, 2004).

En la teoría de redes la internacionalización de empresas se realiza a través de mecanismos integrados de relaciones entre empresas, proveedores y clientes. Esta interacción, que conforma toda una red, se refriere a una forma de penetrar los mercados de manera que las negociaciones interactivas faciliten la comercialización de productos y servicios (Coviello y Munro, 1998). Así pues, las pequeñas y medianas empresas optan por la búsqueda de las oportunidades de negocio en el extranjero por medio de las relaciones empresariales (Johanson y Mattson, 1988), pues su incursión les permite adquirir gradualmente conocimiento experiencial y acceso a nuevas oportunidades de negocio (Johanson y Vahlne, 1990). 
La teoría de la ventaja competitiva de las naciones analiza, entonces, la competencia del mercado y sus oportunidades de inversión, la rentabilidad que tendrá la inversión, la competencia y el apoyo del gobierno con elementos tales como la innovación o la investigación, y así a partir de estos factores se plantea crear una estrategia (Porter, 1991).

En los estudios realizados sobre la internacionalización de empresas colombianas en el sector de alimentos, se planteó que la empresa, al pasar por etapas en la internacionalización, el relacionamiento con los distribuidores, proveedores y clientes, define progresivamente la ventaja competitiva y acelera la expansión (Pineda, Sanabria y Santana, 2011). El éxito de implementar el modelo de relaciones de redes en la estrategia aplica en el caso en el que «la empresa sea realmente miembro de una red y se internacionalice integrándose en redes extranjeras o incrementado su presencia en redes extranjeras a las que ya pertenece» (Piqueras, 1996).

\subsection{Modo de entrada al país destino}

La escuela de economistas de la teoría de proceso de Uppsala afirma que el conocimiento del negocio es progresivo y se perfecciona con la experiencia adquirida en el desenvolvimiento de las operaciones (Johanson y Wiedersheim-Paul, 1975; Johanson y Vahlne, 1977). Las empresas, en su proceso de internacionalización, confían pasar por las cuatro fases al seleccionar un modo de entrada desde exportaciones, licencias, franquicias y filiales hasta sucursales propias; aun así, las relaciones de redes no figuran como determinante para entrar al país destino, si bien las relaciones integradas empresariales abren el mercado internacional e influyen en el modo de entrada y la selección del mercado (Estrategias de mipyme para lograr talla mundial [...], 27 de mayo de 2009). Por tanto, usar modos de internacionalización que requieran optimizar recursos mantiene la baja dependencia en el exterior. 


\subsection{Selección del mercado}

El entorno empresarial clásico se ve como el mercado con proveedores y clientes independientes (Figueroa, 2009). Sin embargo, la empresa que toma la decisión de penetrar mercados en países geográficamente cercanos y con poca distancia psicológica aumenta sus posibilidades de internacionalización (Vernon, 1966). En términos de comercio internacional, los países hacen acuerdos de diferente magnitud, por ejemplo, acuerdos regionales tales como la Alianza del Pacífico (AP); o de complementación económica como el ATPDEA (Andean Trade Promotion and Drug Eradication Act) otorgado por Estados Unidos (Ministerio de Comercio, 28 de agosto de 2018). Así mismo, existen estudios sectoriales como el de ProExport Colombia y SIPPO —el Programa de Promoción de Importaciones(Ferro, Ottens, Van der Linden y Kerver, 2010).

También se encuentran vigentes tratados de libre comercio o TLC como, por ejemplo, el de Colombia con Corea del Sur (Campos, 2016), o entidades como Procolombia, una agencia gubernamental de la rama Ejecutiva del Gobierno de Colombia a cargo de promover las exportaciones, el turismo internacional y la inversión extranjera. Este organismo apoya y asesora a las empresas colombianas en el diseño y la ejecución de la estrategia de internacionalización y presentó en el 2016 la cartilla «TLC Colombia-Corea del Sur: el camino hacia las oportunidades en el mercado asiático». Estos tratados internacionales vigentes son ejes fundamentales para la ejecución de la estrategia de expansión e interconexión con otras latitudes.

\subsection{Enfoque de redes}

La dinámica empresarial es una red de relaciones. El comportamiento de las empresas cambia con el fin de optimizar la penetración a mercados exteriores, de modo que el marketing y la compra se muestran con un rol fundamental en la adquisición de conocimiento y la curva de aprendizaje de una empresa (Dini y Stumpo, 2018). 
En el modelo de redes, las relaciones son el especificador para la selección del mercado, en la cual la empresa que requiera de recursos y proceda de una red logra obtenerlos de acuerdo con la posición que tenga. Las relaciones asiduas de negocios y sociales impactan la selección del mercado (Johanson y Mattson, 1988); mientras que las relaciones directas con los proveedores, distribuidores y clientes ofrecen potencial en la construcción de confianza y compromiso, condiciones previas para la internacionalización.

Figura 2. Estudio de las teorías y factores de internacionalización

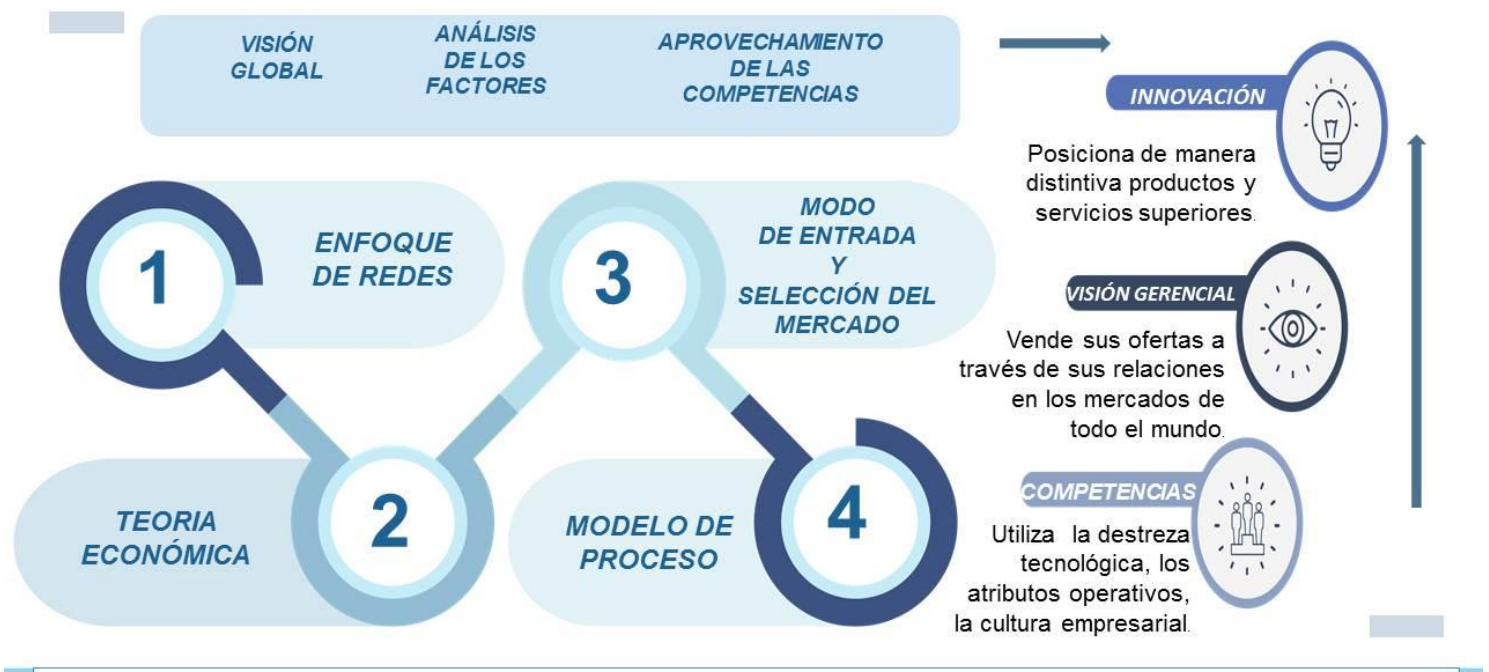

Fuente. Elaboración propia.

Se pude ver en la figura 2 la dinámica comercial, las teorías y los factores de Uppsala —modelo de proceso-, la ventaja competitiva — teoría económica-, las redes —enfoque de redes - y algunos factores -innovación, visión gerencial, competencias - y temas determinantes para la internacionalización — modo de entrada y selección del mercado. Sin embargo, no se trata de implementar un sistema de control interno para afrontar debilidades, pues es indispensable el mantenimiento y la evaluación continua que cause nuevas formas del «cómo», el «cuándo» y el «dónde» hacer (Piqueras, 1996). Tampoco es una respuesta definitiva a las inquietudes sobre las limitantes y libertades de la internacionalización, si bien podría usarse como una herramienta útil en la dinámica comercial. 


\section{Metodología}

Se estableció realizar un estudio del impacto de las relaciones sociales y comerciales en la internacionalización de empresas, a fin de, posteriormente, fijar preguntas de investigación y objetivos.

Por lo tanto, se realizó una revisión documental, literatura de referencia e investigaciones previas, con el propósito de determinar el estado del arte. Se especificó el marco teórico mediante una revisión de las teorías y los factores que definen la evolución del concepto de internacionalización de empresas, con el propósito de situar el relacionamiento de redes como un modo de entrada a mercados externos.

Se utilizó la herramienta analítica check list (Ceballos-Herrera, 2009) para identificar cronológicamente los eventos e interpretar de manera subjetiva la exploración de los casos de estudio. También se realizó una entrevista el día 15 de junio del 2019 al Sr. Albeiro Ayala, gerente administrativo de la empresa Gastro Innova.

La exploración de los casos de estudio presenta elementos conceptuales no estadísticos, bajo los siguientes criterios: condición de la empresa, que sea pyme, debe tener presencia internacional, que sea empresa colombiana y variedad en el modo de entrada. Las fuentes secundarias de información se ampliaron en sus páginas de internet, así como en periódicos, artículos y documentos académicos.

Para el análisis de la información se realizaron tablas y figuras que son congruentes con la investigación. También se llevó a cabo la comparación de las propuestas teóricas en materia de internacionalización de Uppsala, de ventaja competitiva y de redes. En una búsqueda profunda de la importancia del patrón de comportamiento en común se identificaron factores que aceleran el proceso y el impacto de las relaciones de redes en la internacionalización de las pymes. A fin de complementar, como cierre se causan las conclusiones y las recomendaciones. 


\section{Resultados y discusión}

El estudio de las empresas se realiza con base en el impacto de las relaciones sociales y comerciales en la evolución de la internacionalización —selección del mercado y modo de entrada-.

Tabla 1. Características de las empresas Internacionalizadas

\begin{tabular}{|c|c|c|c|}
\hline Características/empresa & A & B & C \\
\hline $\begin{array}{c}\text { Fecha de fundación/año } \\
\text { de exportación }\end{array}$ & $1980 / 1995$ & $1989 / 2003$ & 25 \\
\hline $\begin{array}{c}\text { Número de empleados } \\
\text { Mercados locales } \\
\text { iniciales }\end{array}$ & 6 & 7 & 1 \\
\hline $\begin{array}{c}\text { Mercados } \\
\text { internacionales } \\
\text { actuales }\end{array}$ & $\begin{array}{c}\text { Ecuador, Panamá, } \\
\text { Venezuela, España, } \\
\text { México, Perú, Brasil y } \\
\text { Chile. }\end{array}$ & $\begin{array}{c}\text { Estados Unidos - } \\
\text { Florida-, Canadá } \\
\text { España, Japón. }\end{array}$ & $\begin{array}{c}\text { Panamá, Chile, Venezuela, } \\
\text { Ecuador, Perú }\end{array}$ \\
\hline Modo de entrada & 1 & 3 & 2 \\
\hline
\end{tabular}

Nota. La tabla muestra el lapso de tiempo entre actividad inicial de mercado local e internacional, mercados externos abarcados y número de modos de entrada utilizados.

Fuente. FADE.

Como primera etapa se definen, entre las características, «Fecha de fundación y año de exportación»y «Mercados internacionales actuales» (véase la Tabla 1), en cuanto la evolución de la internacionalización de cada una de las empresas estudiadas. Las empresas A, B y C proyectaron el primer paso a nivel internacional con base en la ventaja competitiva y en la distancia psicológica: «Algunos estudios centran la atención en la influencia de factores inherentes a la propia empresa; otros, sin embargo, consideran la importancia de las características de los países de destino» (Pla, 1999). Aunque el ítem «Mercados locales Iniciales» muestra un mayor posicionamiento de las empresas - $\mathrm{A}$ y $\mathrm{B}$ - , la empresa $\mathrm{C}$ es la que abarca mercado internacional en menor tiempo.

De acuerdo con las características de las empresas estudiadas, se puede decir que la toma de decisiones ha sido racional (Trujillo-Dávila, Rodríguez-Ospina, Guzmán-Vásquez y 
Becerra-Plaza, 2006). La información que obtuvo cada una de las empresas de los resultados de la evaluación de la plaza local — segunda etapa-y de los resultados de la evaluación para la expansión - tercera etapa- hicieron del conocimiento experiencial una incubadora para la internacionalización (Johanson y Wiedersheim-Paul, 1975).

Figura 3. Esquema del proceso de internalización de las empresas A, B y C

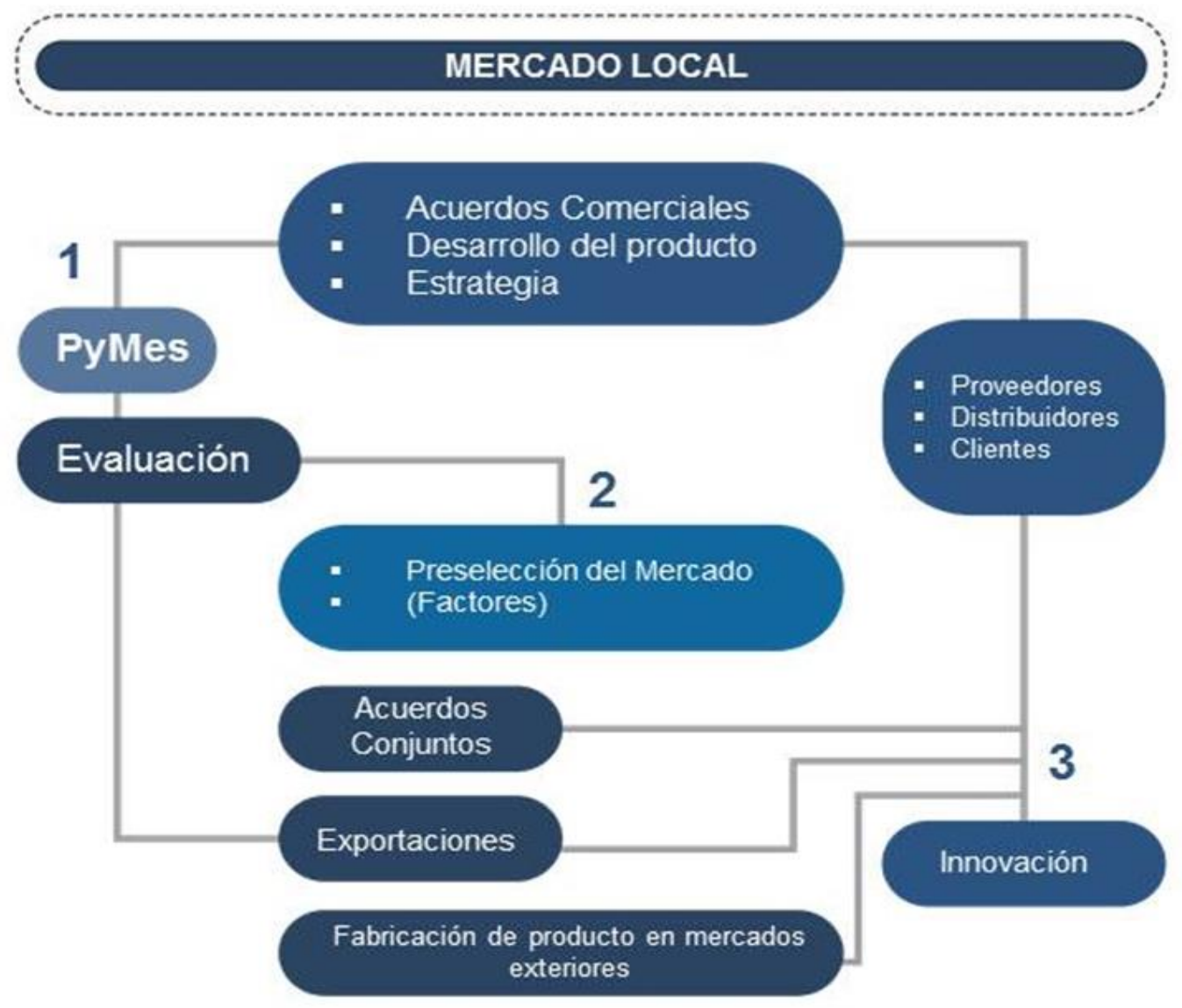

Nota. La figura representa tres momentos del proceso de internacionalización: 1) actividad local, 2) selección del mercado, y 3) modo de entrada para la expansión internacional.

Fuente. Elaboración propia.

Respecto a los planteamientos generales sobre habilidades que se adquieren en el proceso de internacionalización de las empresas, como, por ejemplo, la visión global, el compromiso gerencial y la postura competitiva, el conocimiento del mercado se da de 
manera gradual. Este gradualismo experiencial se presenta a la luz del método Uppsala como cambios en las prácticas comerciales (Johanson y Vahlne, 1977). Con base en el enfoque de red, Cardozo, Chavarro y Ramírez (2013) llevaron a cabo estudios empíricos sobre la internacionalización de las pequeñas empresas de software en Bogotá y encontraron que las relaciones de redes tienen un impacto serio en la selección del mercado, así como en el modo de entrada.

La figura 3 muestra el esquema de la gestión del relacionamiento comercial con proveedores, distribuidores y clientes, e involucra tres momentos en el proceso de la internacionalización de las empresas estudiadas: inicia antes de la primera exportación momento 1, actividad local—, luego adquiere habilidades y análisis de los factores momento 2, selección del mercado-, y, finalmente, obtiene los resultados de la evaluación para implementar la estrategia de expansión internacional - momento 3, selección del modo de entrada-

Tabla 2. Factores que aceleran el proceso de internacionalización

\begin{tabular}{|c|c|c|c|}
\hline Factores/empresa & $\mathbf{A}$ & B & C \\
\hline 1. Visión global & Sí & Sí & Sí \\
\hline 2. Relaciones de redes & Sí & Sí & Sí \\
\hline $\begin{array}{l}\text { 3. Compromiso gerencial hacia la } \\
\text { internacionalización }\end{array}$ & $\begin{array}{l}\text { Mercados locales y } \\
\text { cercanos }\end{array}$ & $\begin{array}{l}\text { Mercados locales y } \\
\text { lejanos }\end{array}$ & $\begin{array}{l}\text { Mercado locales y } \\
\text { cercanos }\end{array}$ \\
\hline 4. Experiencia internacional de gerentes & NO & Sí & Sí \\
\hline $\begin{array}{l}\text { 5. Fuentes principales de creación de } \\
\text { valor }\end{array}$ & $\begin{array}{l}\text { RSE (responsabilidad social } \\
\text { empresarial) }\end{array}$ & $\begin{array}{l}\text { Diversificación de } \\
\text { producto y tecnología }\end{array}$ & $\begin{array}{l}\text { Diversificación de } \\
\text { producto y tecnología }\end{array}$ \\
\hline $\begin{array}{l}\text { 8. Distancia psicológica/estrategia } \\
\text { internacional }\end{array}$ & $\begin{array}{l}\text { Mercados locales y } \\
\quad \text { cercanos }\end{array}$ & $\begin{array}{l}\text { Mercados locales, } \\
\text { cercanos y lejanos }\end{array}$ & $\begin{array}{l}\text { Mercados locales y } \\
\quad \text { cercanos }\end{array}$ \\
\hline $\begin{array}{l}\text { 6. Selección de grupo de clientes, } \\
\text { orientación al cliente y relación con los } \\
\text { clientes }\end{array}$ & Sí & Sí & Sí \\
\hline 7. Flexibilidad estratégica & Sí & Sí & Sí \\
\hline
\end{tabular}

Fuente. FADE. 
La tabla 2 expone la relación de los factores que dan celeridad al proceso de internacionalización de las empresas estudiadas. En el análisis, la empresa C entra al mercado internacional debido al conocimiento y la experiencia adquiridos por el gerente ( Federico et al., 2009). Así, la primera exportación a Panamá (véase la Tabla 1) se da por los constantes viajes; específicamente el gerente de esta empresa nota que en Panamá los productos que se utilizaban se producían bajo diferentes procesos y eran importados desde Estados Unidos y Brasil.

Al regresar a Colombia, el gerente presenta una propuesta de venta del producto a la compañía de Panamá, resaltando la experiencia en el segmento, la cercanía al país y el costo competitivo en fletes. La selección del mercado y el modo de entrada lo señala la visión global del gerente, la intensión exportadora y la propuesta de valor; sin duda, los factores mencionados son decisivos para la internacionalización.

Las empresas estudiadas abarcan mercados internacionales al mismo tiempo que carecen de recursos. En las evaluaciones toman el control de los activos y usan los medios disponibles para las estrategias con los distribuidores extranjeros que brindan ventajas locales relacionadas no solo con la actividad comercial internacional, sino también con la recopilación de inteligencia de mercado; forjando así, vínculos con contactos extranjeros clave, profundizando las relaciones dentro de los mercados existentes y cultivando nuevos segmentos de compradores, de modo que hacen factible la expansión internacional (Knight y Cavusgil, 2004; Johanson y Mattson, 1988).

Las barreras de entrada que enfrentan las pymes, como, por ejemplo, las tasas de cambio, las tarifas operacionales y de transporte, la capacitación del recurso humano, la capacidad de producción y la competencia en el mercado global, se convierten en retos para mejorar la postura competitiva, la innovación, la tecnología y los procesos en gestión de políticas de desarrollo industrial. En términos generales, la creación de bloques solidarios posibilita a las empresas internacionalizarse. Conviene subrayar que el mayor indicador de oportunidades que delimita la selección del mercado es la búsqueda de participación en redes. 
Gastón Acurio, chef, escritor y hombre de negocios, expresa que el proceso de internacionalización del restaurante de comida peruana, empresa que inicio bajo la marca Astrid \& Gastón, se debe al conocimiento previo que proporcionan las relaciones de redes:

\begin{abstract}
Es importante tratar de buscar un socio local por que uno llega con el conocimiento del servicio o producto que uno quiera colocar. Sin embargo, hay conocimiento local que no necesariamente uno lo va a poder entender, lidiar con legislación local, saber escoger la ubicación adecuada, el tiempo correcto, proveedores locales, el tema laboral local, la sociedad local, los desafíos de la sociedad, en términos de qué les gusta que no les gusta, buscar un socio local con experiencia que tenga comprobado éxito en la industria a la que uno se dedique (no un socio inversionista que de repente no conoce el negocio ni el lugar que uno quiera abrir); ese es el paso ideal para tener éxito (un socio inversionista que no te aporta conocimiento de la industria, quizás no es el más adecuado) un socio que tenga experiencia en la industria, esto es lo más importante incluso más allá del capital que te pueda aportar. (Mundo empresarial, 17 de julio de 2014)
\end{abstract}

Tabla 3. Presentación del impacto de las relaciones de redes en la internacionalización

\begin{tabular}{|c|c|c|c|}
\hline & Inicios de la pyme & $\begin{array}{l}\text { Primer modo de } \\
\text { entrada }\end{array}$ & Modos de entradas siguientes \\
\hline $\mathbf{A}$ & $\begin{array}{l}\text { Equipo fundador }- \text { dos- } \\
\text { Empresa familiar, } \\
\text { universitarios. } \\
\text { Sin experiencia internacional } \\
\text { personal sin experiencia en sus } \\
\text { áreas. }\end{array}$ & $\begin{array}{l}\text { Franquicia: crearon } \\
\text { relaciones con socios en } \\
\text { Ecuador. }\end{array}$ & $\begin{array}{l}\text { Franquicia: incremento el compromiso en redes ya } \\
\text { establecidas: diez puntos de venta en Ecuador. }\end{array}$ \\
\hline $\mathbf{B}$ & $\begin{array}{l}\text { Equipo fundador -ocho- } \\
\text { Socios, profesionales. } \\
\text { Con experiencia internacional. } \\
\text { Personal con experiencia en sus } \\
\text { áreas. }\end{array}$ & $\begin{array}{l}\text { Filial: crearon relaciones } \\
\text { con socios en Estados } \\
\text { Unidos. }\end{array}$ & $\begin{array}{l}\text { Franquicia: incrementó el compromiso en redes ya } \\
\text { establecidas. } \\
\text { Exportación directa: participación de la Feria } \\
\text { Internacional Foodex, Japón. }\end{array}$ \\
\hline $\mathbf{C}$ & $\begin{array}{l}\text { Equipo fundador -cuatro- } \\
\text { Empresa familiar y socios, } \\
\text { profesionales. } \\
\text { Con experiencia internacional. } \\
\text { Personal sin experiencia en sus } \\
\text { áreas. }\end{array}$ & $\begin{array}{l}\text { Exportación } \\
\text { esporádica: crean } \\
\text { relación con socio; la } \\
\text { exportación se dio } \\
\text { gracias en un viaje a ese } \\
\text { país del gerente } \\
\text { administrativo y } \\
\text { financiero. }\end{array}$ & $\begin{array}{l}\text { Exportaciones directas: Participación en la Feria } \\
\text { Internacional Avipla, se convierte en el proveedor } \\
\text { estratégico. } \\
\text { Fusión: cada empresa en su país origen, como } \\
\text { proveedores regionales en sus respectivas } \\
\text { industrias, desarrolla estrategias de productos en } \\
\text { conjunto. }\end{array}$ \\
\hline
\end{tabular}

Fuente. FADE. 
La estrategia de internacionalización inicial y para la expansión de la empresa A es la franquicia. Este modo de internacionalización es el contrato reconocido por fortalecer la competencia de pymes respecto a las grandes compañías, dado que los resultados del crecimiento son continuos. En este formato el franquiciador - empresa A- se encargó del artículo, es decir, de la marca y el mercadeo de los productos, mientras el franquiciado socio en México- asumió las responsabilidades de la producción y las ventas.

La estrategia empleada por la empresa B es en la modalidad de entrada filial: elabora un contrato atípico de franquicia e incluye el anexo denominado «arriendo de operación», en el cual se especifica que el socio inversionista -Estados Unidos- les arrienda el establecimiento de comercio y de la filial misma. El crecimiento continuo permitió a la empresa B la apertura de cuatro mercados más y los modos de entrada siguientes fueron la exportación directa y la franquicia.

Por su parte, la empresa C para la estrategia inicial utiliza el modo de entrada exportación indirecta y luego usa la exportación directa y la fusión; la exportación probablemente es la estrategia más simple para internacionalizarse. La primera exportación indirecta de la empresa C es ocasional; esta modalidad se realiza mediante un intermediario independiente que se hace cargo de todos los trámites. La siguiente exportación es directa y se refiere a que el gerente mismo se encarga del contacto directo con el cliente, de la logística y la tramitología de la exportación de los productos. Posterior a la consolidación de las relaciones con su socio comercial, la empresa utiliza la estrategia de la «fusión». Esta modalidad es la unión entre dos empresas para crear una de mayor tamaño, y es de alto grado con miras a la expansión internacional de las pymes, pero sigue siendo un riesgo para el inversionista.

De acuerdo con Trujillo-Dávila, Rodríguez-Ospina, Guzmán-Vásquez y Becerra-Plaza, (2006), «en los negocios internacionales a veces es necesaria alguna forma de compromiso social para poder establecer las bases formales antes de comenzar con las negociaciones» (p. 25). En el análisis del impacto de las relaciones en la internacionalización de las empresas 
estudiadas, las relaciones comerciales tuvieron mayor influencia en la selección del mercado mientras que las relaciones sociales tuvieron impacto en la expansión (véase la Tabla 3).

Figura 4. Perspectiva de internacionalización para pyme

\section{RELACIONES DE REDES}

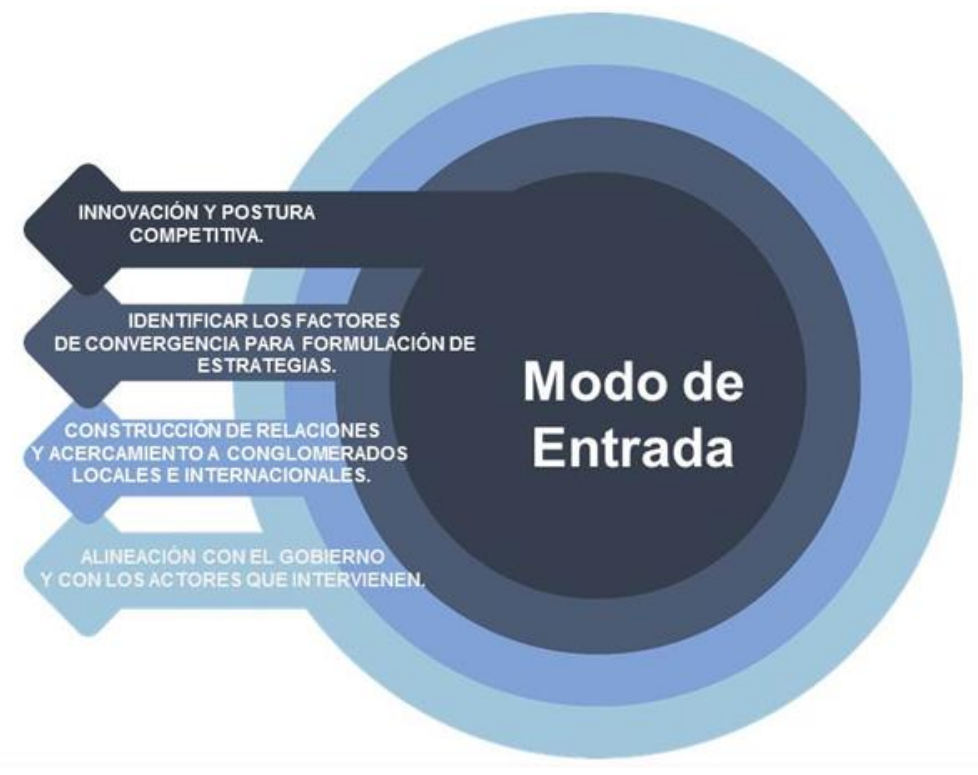

Fuente. Elaboración propia.

Para finalizar, la academia relaciona el modo de entrada como costes de transacción — teoría de la internalización-. Hay quienes refutan esta idea, pues la posición competitiva de la empresa a través de esquemas de participación, al identificar factores de convergencia alineados con el gobierno y con los actores que intervienen y tienen que interactuar, consolida las relaciones con los socios extranjeros. Las relaciones de redes impactan la selección del mercado y se convierten en el modo de entrada de alto grado. De esta manera se proporciona la perspectiva de internacionalización (véase la Figura 4) para la empresa Gastro Innova. La postulación de las teorías de internacionalización de las empresas evalúa dos formas del mercado: las medidas externas - exportaciones - y las medidas internas inversión directa-. 
De lo primero que se debe ser consciente es que no es lo mismo exportar que internacionalizar, exportar es solo una de [las] muchas formas que una empresa ingresa a la dinámica del comercio global, mientras que internacionalizar es el conjunto de acciones que lleva a cabo la empresa para operar y generar un negocio de continuo crecimiento en uno o varios mercados extranjeros, bajo una estrategia. (Internacionalmente, 15 de julio de 2016)

\section{Recomendaciones}

Antes del COVID-19, la globalización, la hiperconexión, el internet móvil y el fácil acceso a los productos y los servicios, Colombia era una de las economías más dinámicas de América Latina; el Banco de la Republica proyectó una meta de crecimiento del 3\% al 2020 para Colombia (Fondo Monetario Internacional, 8 de marzo de 2019). El principal objetivo en ese momento era disminuir el desempleo y la estabilidad de la inflación, no obstante, la llegada del COVID-19 al país, la devaluación del precio del petróleo y las políticas para cesar el contagio, como, por ejemplo, el distanciamiento social, redujeron significativamente el suministro de la mano de obra que opera la gran parte de la economía - comercio, turismo, entretenimiento-, lo que ha generado costos importantes para la producción (Hevia y Neumeyer, 2020).

Hoy, el COVID-19 supone un momento disruptivo: distanciamiento social, home office, brecha digital, aceleración de procesos, trabajo en equipo remoto, etc.; las dimensiones globales de la crisis declaran efectos permanentes de deterioro en la económica mundial (Miller y Rivas, 2020).

La Comisión Económica para América Latina y el Caribe (Cepal) presenta una de las características de la crisis:

Cooperación internacional: la salida de la crisis dependerá de la fortaleza económica de cada país, por lo tanto, dadas las asimetrías entre los países desarrollados y en desarrollo, el papel de la ONU, el FMI y el Banco Mundial será esencial para garantizar el acceso al financiamiento, sostener el gasto social y mantener la actividad económica con medidas innovadoras («out of the box»).

(Bárcena, 2020) 
Hacer frente a la crisis requirió escalar las capacidades nacionales y regionales, principalmente, en la producción y la provisión de bienes de primera necesidad. En Colombia se tomaron rápidamente algunas medidas macroeconómicas para hacer frente a la emergencia:

- El Fondo Nacional de Garantías y el Fondo para el Financiamiento del Sector Agrícola habilitó los recursos que permitieran avalar la disponibilidad de crédito para las micro, pequeñas y medianas empresas. Esto hizo posible que los sectores fundamentales para la economía o muy afectados por la crisis también pudieran contar con liquidez (Mincomercio, 2 de julio de 2020).

- Encendida la alarma sanitaria a nivel mundial, el cambio del calendario fiscal retrasó los pagos de impuestos, mantuvo los alquileres, el gobierno tomó la decisión de no cobrar los pagos de aportes pensionales a las empresas — que se tendrán que pagar al final de la vida laboral- y asumió las nóminas de las pymes por tres meses (Sánchez, 10 de abril de 2020).

«Después del COVID-19», en la nueva normalidad, los sectores agrarios, de cuidado personal y de salud, food procesing \& retail y servicios médicos son potencialmente ganadores del COVID-19. La crisis sanitaria generó varias dificultades para los empresarios y los emprendedores, así como abrió las puertas a la reinvención; los sectores mencionados enfrentan la situación recurriendo a una serie de estrategias empresariales alineadas al $e$ commerce, a la regionalización y al desarrollo de habilidades que afiancen formas alternativas de intercambio comercial.

Por tanto, sin violentar las medidas de salubridad establecidas por los gobiernos nacionales, el compromiso aumentó en las relaciones con los proveedores, los distribuidores, los clientes y colaboradores, y dinamizó la estructura organizativa; ahora las empresas acuden a los valores corporativos y al compromiso del personal que las integran para mantener la operación (González-Díaz y Flores-Ledesma, 2020). 
Plagados de incertidumbre, riesgo y con recursos relativamente limitados, el gran desafío de las pymes será la postura competitiva e innovadora, así como la visión proactiva en la búsqueda de nuevos mercados en el escenario actual de compleja convivencia con el virus.

\section{Conclusiones}

La internacionalización de las pymes inicia por un sistema de comercialización exploratorio, bajo en riesgos y costos. El conocimiento del mercado es fundamental en el propósito de adaptarse a cada público objetivo, momento en que las relaciones de redes y el comercio internacional se ven muy ligados, ya que se necesita estar presente en las redes si se busca presencia internacional.

La experiencia previa en la dinámica comercial internacional es un factor importante en el «perfil del gerente». Activarse en el comercio internacional es definir el comportamiento y la actitud hacia la idea de internacionalización; en esos términos, decidir si es positivo o no para la empresa incursionar en mercado extranjero.

El crecimiento progresivo de las pymes exploradas es evidente, el inicio de las actividades en el mercado local causa y consolida las relaciones de negocio y las relaciones sociales que potenciarán la expansión internacional.

La estructura financiera es la situación más común que pone en riesgo a la mayoría de pymes, por consiguiente, la mayoría de veces se supone, en teoría, que a mayor tamaño de la organización mayor es el capital que le permitirá posicionarse rápidamente y establecer operaciones en el mercado internacional.

Adquirir conocimiento parece un problema en el proceso de internacionalización, pero el problema es cómo se administra la carencia de ese conocimiento; un incremento en la especialización en comercio internacional aumenta la confianza y las posibilidades de generar compromiso en las relaciones de redes. 


\section{Referencias}

Aladi. (2011). Oportunidades comerciales Ecuador-Colombia. Recuperado de https://bit.ly/3fZibV9

Amestoy, L. H. (2009). Políticas para las mipymes frente a la crisis. Conclusiones de un estudio comparativo de América Latina y Europa. OIT. Recuperado de https://bit.ly/3d17oba

Bárcena, A. (3 de abril de 2020). Coyuntura, escenarios y proyecciones hacia 2030 ante la presente crisis de COVID-19. Cepal. Recuperado de https://bit.ly/3mAySHI

Bauman, Z. (2016). La globalización: consecuencias humanas. Ciudad de México: Fondo de Cultura Económica.

Cámara de Comercio de Bogotá. (2009). Informe sobre las causas de la liquidación de empresas en Bogotá. Bogotá: CCB.

Campos, S. V. (2016). ¿Cómo va la Alianza del Pacífico frente a Asia? Bogotá: Technical Report.

Cardozo, P. P.; Chavarro, A.; Ramírez, C. A. (2013). Las pymes bogotanas y sus procesos de internacionalización-Primer balance. Revista Panorama, 1(3), 24-42. DOI: https://doi.org/10.15765/pnrm.v1i3.265

Ceballos-Herrera, F. A. (2009). El informe de investigación con casos de estudio. Magis, Revista Internacional de Investigación en Educación, 1(2), 413-423. Recuperado de https://bit.ly/3futVrT

Coviello, N.; Munro, H. (1998). Network relationships and the internationalization process of small firms. International Business Review, 6(4), 361-386. DOI: https://doi.org/10.1016/S0969$\underline{\text { 5931(97)00010-3 }}$

Dini, M.; Stumpo, G. (Coords.). (2018). Mipymes en América Latina: un frágil desempeño y nuevos desafíos para las políticas de fomento. Santiago: Comisión Económica para América Latina y el CaribeCepal.

Drucker, P. (1993). La sociedad poscapitalista. Argentina: Editorial Sudamericana.

Estrategias de mipyme para lograr talla mundial; representantes del sector se dieron cita en Cali. (27 de mayo de 2009). Portafolio. Recuperado de https://bit.ly/3fVIMqu

Federico, J. S.; Kantis, H. D; Rialp, A. R. (2009). Does entrepreneurs' human and relational capital affect early internationalization? A cross-regional comparison. European Journal of International Management, 3(2), 199-215. DOI: https://doi.org/10.1504/EJIM.2009.024322

Ferro, G.; Ottens, B. J.; Van der Linden, M.; Kerver, K. (2010). Fortalecimiento de la Capacidad Comercial hacia los Países EFTA: Inteligencia de Mercados para Colombia - Alimentos Procesados. Osec Zurich, Business Network Switzerland. Recuperado de https://bit.ly/39WnDnR

Figueroa, A. M. (2009). Aproximación al proceso de internacionalización de las empresas: el caso colombiano. Bogotá: Universidad del Rosario. 
Fondo Monetario Internacional. (8 de marzo de 2019). Colombia: declaración final al término de la misión del artículo IV (año 2019). Recuperado de https://bit.ly/3mvS3m0

Giddes, A. (2000). Un mundo desbocado: los efectos de la globalización en nuestros días. Madrid: Taurus. Recuperado de https://bit.ly/3uyB9WB

González-Díaz, R. R.; Flores-Ledesma, K. N. (2020). Cultura organizacional y sustentabilidad empresarial en las pymes durante crisis periodos de confinamiento social. Revista Internacional Multidiciplinaria, 1(1), 28-41. DOI: https://doi.org/10.46785/ciidj.v1i1.40

Hernández-Pereira, R. (2017). Impacto de las capacidades de internet en la gestión, la operativa internacional y la internacionalización de las PYMES exportadoras. Ocho estudios de caso en Costa Rica. (Tesis de doctorado). Universidad de Sevilla, Sevilla. Recuperado de https://bit.ly/3wCVzzH

Hevia, C.; Neumeyer, A. (20 de marzo de 2020). Un marco conceptual para analizar el impacto económico del COVID-19 y sus repercusiones en las políticas. PNUD América Latina y el Caribe. Serie de documentos de política pública. Recuperado de https://bit.ly/3dHeYH6

Internacionalmente. (15 de julio de 2016). Mercados internacionales: descubre las mejores formas de entrada. Recuperado de https://bit.ly/3d1H40v

Irineo, G. P.; Morales, L. E.; Mora, J. P.; Montemayor, J. G. (2015). Revisión teórica de los factores críticos de éxito que influyen en la internacionalización de las empresas del sector de alimentos procesados. Ponencia presentada en el XXI Congreso Internacional de Contaduría, Administración e Informática. Universidad Estatal de Sonora, Sonora, México, 5-7 de octubre. Recuperado de https://bit.ly/31YbtXj

Johanson, J.; Mattson, L. (1988). Internationalization in industrial systems-a network approach. En M. Forsgren; U. Holm; J. Johanson (eds.) Knowledge, networks and power. (111-132). Londres: Palgrave Macmillan. DOI: https://doi.org/10.1057/9781137508829 5Recuperado

Johanson, J.; Vahlne, J.-E. (1977). The internationalization of the firm-A Model of knowledge development and increasing foreign market commitments. Journal of International Business Studies, 8(1), 2332. DOI: $\underline{\text { https://doi.org/10.1057/palgrave.jibs.8490676 }}$

Johanson, J.; Vahlne, J.-E. (1990). The mechanism of internationalization. International Marketing Review, 7(4). DOI: https://doi.org/10.1108/02651339010137414

Johanson, J.; Wiedersheim-Paul, F. (1975). The internationalization of the firm-four Swedish cases. Journal of International Business Studies, (12), 305-322. DOI: https://doi.org/10.1111/j.1467$\underline{6486.1975 . t b 00514 . x}$

Knight, G. A.; Cavusgil, S. T. (2004). Innovation, Organizational Capabilities, and the BornGlobal Firm. Journal of International Business Studies, 35(2), 124-141. DOI: https://doi.org/10.1057/palgrave.jibs.8400071

Miller, J. C.; Rivas, M. D. (2020). Implicaciones económicas de la pandemia por COVID-19 y opciones de política. Notasestratégicas, (81). Recuperado de https://bit.ly/3t6MTzp 
Ministerio de Comercio (Ministerio de Comercio, Industria y Turismo). (28 de agosto de 2018). Exportadores de alimentos procesados a Estados Unidos reciben capacitación que les ayudará a aprovechar más el TLC. Recuperado de https://bit.ly/31VKagg

Ministerio de Comercio, Industria y Turismo (Mincomercio). (2 de julio de 2020). Garantías por un billón de pesos para mipymes de los sectores de restaurantes, entretenimiento y alojamiento, afectadas por el COVID-19. Recuperado de https://bit.ly/31ZBXHS

Mundo empresarial. (17 de julio de 2014). Caso chef Gastón Acurio [video]. Youtube. Recuperado de https://bit.ly/3s040GM

Pineda, A.; Sanabria, V.; Santana, A. (2011). Internacionalización de las empresas colombianas casos de estudio: Hamburguesas El Corral y Pan Pa' Ya! (tesis de grado). Universidad Del Rosario. Bogotá, Colombia. Recuperado de https://bit.ly/31ZeczO

Piqueras, J. J. (1996). El por qué, el cómo y el dónde de la internacionalización de la empresa. Revista Austariana de Economía, (6). 41-62.

Pla, J. (1999). Filiales y entrada en los mercados internacionales. Factores determinantes. Revista de Economía Aplicada EA, 7(20), 29-51.

Porter, M. E. (1991). La ventaja competitiva de las naciones. Revista Facetas, (91), 1-91. Recuperado de https://bit.ly/3uy6qZU

Procomer. (2017). Retos y oportunidades para la oferta de alimentos diferenciados. Costa Rica: Agencia de Gobierno.

Propaís. (27 de mayo de 2009). Encuentro por la transformación productiva e internacional de las pymes. Recuperado de https://bit.ly/3s3C3sw

Puerto-Becerra, D. P. (2010). La globalización y el crecimiento empresarial a través de estrategias de internacionalización. Pensamiento \& Gestión, (28), 171-195.

Rojas-Villa, L. (2015). Neoliberalismo en América Latina. Crisis, tendencias y alternativas. Asunción: Ministerio Federal de Cooperación Económica y Desarrollo de Alemania (BMZ), Consejo Latinoamericano de Ciencias Sociales (CLACSO).

Sánches, A. M. (10 de abril de 2020). Pago de nómina de Pymes y suspensión de aportes a pensión, medidas del Gobierno. La República. Recuperado de https://bit.ly/2Q9CNPy

Trujillo-Dávila, M. A.; Rodríguez-Ospina, D.; Guzmán-Vásquez, A.; Becerra-Plaza, G. (2006). Perspectivas teóricas sobre internacionalización de empresas. Bogotá: Universidad del Rosario.

Vernon, R. (1966). International Investment and International Trade in the Product Cycle. The Quarterly Journal of Economics, 80(2), 190-207. DOI: https://doi.org/10.2307/1880689

Viscarri-Colomer, J. V. (2011). Modelo de creación de valor para el cliente. Ponencia presentada en el Congreso Internacional de Contaduría, Administración e Informática. Ciudad Universitaria, México D.F., México, 5-7 de octubre. Recuperado de https://bit.ly/3t2xdgD 